\title{
EL PROYECTO DE LAS ESCUELAS DE BARRIADA Y SU DESARROLLO (1919-1938). UNA EPOPEYA EN LA HISTORIA DE LA EDUCACIÓN DE BIZKAIA
}

por Matte Paliza Munduate. Bilbao y Vitoria-Gasteiz: Servicio Central de Publicaciones del Gobierno Vasco: Diputación Foral de Bizkaia, 2015, 227 páginas (versión castellano) + 225 páginas (versión euskera). ISNN: 978-84-457-3361-5 (EJAZN/SCPGN); 978-84-7752-568-4 (BFA/DFB)

El libro El proyecto de las ESCUELAS DE BARRIADA y su desarrollo (1919-1938). Una epopeya en la historia de la educación de Bizkaia nos describe la historia de un proyecto renovador y referente por lo que respecta a la enseñanza rural y la arquitectura escolar, que fue desarrollado por la Diputación de Bizkaia, en colaboración con los consistorios locales, vecinos y padres de los alumnos de más de 100 barriadas de Bizkaia. Teniendo en cuenta que la escolarización está directamente relacionada con la creación de escuelas, y que éstas, históricamente, se han potenciado sobre todo en los núcleos urbanos, no debe de extrañar que la enseñanza en los barrios rurales haya sido tratada muchas veces como un tema secundario. Así pues, hay que darle la bienvenida a este libro de Maite Paliza Munduate. Tenemos que destacar que este proyecto, que fue pionero en la alfabetización de las zonas rurales, despertó el interés en otros puntos del Estado e incluso en Iberoamérica.

Para la elaboración de la presente monografía, Maite Paliza, catedrática de la Historia del Arte de la Universidad de Salamanca, toma como referentes sobre todo dos publicaciones: el monográfico Las Escuelas de Barriada de Vizcaya de Pedro Zufia, inspector de estas escuelas, publicado en 1930, y el libro Educación y escuelas de barriada de Bizkaia. Escuelas y Autonomía 1989-1936, editado por la Diputación de Bizkaia en 1987. Ha tenido en cuenta también otras publicaciones especializadas, sobre todo a la hora de analizar el proyecto desde el punto de vista arquitectónico. A partir de estas bases documentales, hay que destacar el trabajo de 
documentación realizado por la autora, que aporta una serie de planos, fotografías e informaciones inéditas de un gran valor para investigaciones posteriores.

Este libro describe un proyecto pionero que durante las décadas de los años veinte y treinta del siglo pasado contribuyó a disminuir la tasa de analfabetismo del territorio vizcaíno. Así, a partir de 1919, la Diputación de Bizkaia potenció este modelo alternativo educativo, que fue la primera tentativa, dentro de un modelo oficial, en la que se impartía la enseñanza en euskera. Mucho se ha discutido sobre la aportación de las escuelas de barriada a la causa de la cultura nacionalista; la realidad es que hay que analizar el desarrollo de estas escuelas encuadrándolas en distintos momentos políticos. Si en su creación, y en los primeros años de su desarrollo, el auge de la ideología nacionalista tuvo una importancia considerable, en 1921, como consecuencia del triunfo de la Liga Monárquica, se dio un cambio político y en consecuencia de orientación lingüística, suprimiendo la utilización del euskara en los centros docentes. Aunque unos años después los cambios políticos acaecidos en la II República sirvieron para potenciar la enseñanza y la creación de nuevos centros educativos, el golpe militar de 18 de julio de 1936 truncó todo proyecto renovador y mediante la Orden de 18 de abril de 1938, las Escuelas de Barriada de Bizkaia y las Rurales de Gipuzkoa pasaron a depender del Estado, convirtiéndose en escuelas nacionales.

El volumen está organizado en dieciséis capítulos y dos prólogos, escritos estos por los actuales responsables políticos y administrativos de las instituciones editoras. Aunque desde mi perspectiva estos capítulos se podían haber organizado en tres grandes apartados (uno, primero, describiendo la situación de la enseñanza rural; un segundo apartado desarrollando el proyecto en su doble dimensión pedagógica y arquitectónica, así como en relación con el personal, incidiendo en el papel del profesorado, inspección y alumnado; y una tercera parte analizando el final de este proyecto), es evidente que la estructuración presente tiene la ventaja de facilitar el acercamiento a cada uno de los temas enumerados.

La autora, en un primer capítulo, hace una breve introducción en la que señala que esta experiencia educativa despertó una gran expectación en otros lugares, sea desde la vecina Diputación de Gipuzkoa, que llegó a impulsar un proyecto semejante entre los años 1931-1936, o desde 
Uruguay. Por lo que respecta a los protagonistas de esta magna empresa, Paliza destaca sobre todo a los inspectores Luis Elizalde y Pedro Zufia, y al arquitecto provincial Pedro Basterra. El segundo capítulo nos habla de la situación de la enseñanza antes de la creación de estas escuelas y de las transformaciones acaecidas en el sistema educativo estatal, como resultado de una legislación específica.

En el tercer capítulo, la autora enumera, en particular, las características más importantes de este proyecto, destacando la figura del diputado Juan Gallano, que presentó ante la corporación provincial la moción para difundir la enseñanza en las zonas apartadas de la geografía vizcaína. En el reglamento aprobado por la Diputación se establecía que era competencia de esta corporación la creación, distribución, organización y administración de los centros docentes. Pero como la creación debía ser ratificada por la Universidad de Valladolid, hubo problemas como consecuencia de que la legislación no otorgaba competencias en materia de enseñanza a las diputaciones, sobre todo por el intento de implantar el bilingüismo. Para finalizar este capítulo se señala cual era la coyuntura de la época, que coincidió con el triunfo del nacionalismo en las elecciones de 1917. Sin embargo, en los años posteriores este nacionalismo sufrió un revés siendo superado por la Liga Monárquica. Se hace también hincapié en la figura de Eduardo Landeta Aburto, que fue vocal de Instrucción pública del Ayuntamiento de Bilbao y estuvo al frente de la Sección de Primera Enseñanza de la Sociedad de Estudios Vascos entre 1918 y 1930. En el cuarto capítulo es la realidad rural de Bizkaia, con una situación económica precaria, con numerosos barrios y caseríos aislados, con una complicada orografía y una deficiente red de caminos y carreteras, la que se aborda. A lo anterior se añade la cuestión del idioma: en las zonas rurales el euskera era la única lengua que conocían la mayoría de los niños y niñas, aunque, sin embargo, era una gran desconocida para la mayoría de los maestros y maestras.

Los capítulos siguientes, desde el cinco al catorce, describen como fue la creación de los nuevos centros y la evolución del proyecto: el proceso de la creación de las primeras escuelas a partir de 1921 y de las que se crearon en los años posteriores, en los cuales se fueron construyendo una media de 10 escuelas cada año; la labor de los técnicos de la Diputación responsables de la materialización del proyecto, citando entre otros al 
arquitecto provincial Diego Basterra y Berasategui y al técnico del servicio de Instrucción Luis Eleizalde Breñosa, figura destacada de la cultura vasca de la época; o también las características del emplazamiento de los edificios y de los solares en los que se ubicaban las escuelas, lo que en diversas ocasiones supuso además una importante implicación social.

Teniendo en cuenta que una de las líneas de investigación que más ha trabajado Maite Paliza ha sido la de la arquitectura, no es de extrañar que sea el capítulo décimo, el relativo a las edificaciones escolares, uno de las más trabajados. Se describen los prototipos diseñados por el arquitecto provincial y sus características, haciendo hincapié en las exigencias pedagógicas y de higiene de la época, sobre todo en la correcta ventilación e iluminación de los edificios, como se puede observar en la interesante planimetría que se incorpora. Los planos y las fotografías que ilustran este capítulo nos sirven para ver la realidad diversa de estas escuelas. Desde mi punto de vista, el capítulo que trata de la construcción de las escuelas y de los obstáculos que surgieron, podría haberse incluido en este apartado.

El capítulo once nos habla de su financiación, señalando cómo en la década de los años veinte, las arcas municipales de la mayoría de los ayuntamientos vizcaínos estaban en una situación muy precaria, que no permitía afrontar los gastos que exigían estas construcciones. Así, lo habitual era que los consistorios aportasen el terreno y que los propios vecinos colaboraran en la construcción del inmueble en la medida de sus posibilidades.

En la parte final de este libro, desde el capítulo trece hasta el dieciséis, se describen los planteamientos pedagógicos y administrativos de las escuelas. Al caso, en el capítulo trece se nos muestran, mediante fotografías, el mobiliario y el equipamiento de que dispusieron estas escuelas, acorde con la renovación pedagógica; la biblioteca circulante del Maestro y el empleo del cinematógrafo. En este capítulo se cita también la labor de la Caja de Ahorros Vizcaína que aporto una biblioteca a cada una de las escuelas.

El capítulo catorce nos señala como este proyecto no solo contemplaba la docencia teórica, encaminada a erradicar el analfabetismo, sino que incluía y potenciaba actividades al aire libre, tanto juegos infanti- 
les durante los recreos como clases específicas de gimnasia, para mejorar la salud y el desarrollo de los estudiantes, así en algunas escuelas se construyeron frontones. También mediante este proyecto se potenciaron actividades educativas relacionadas con el sector agrícola-ganadero: en bastantes casos se procedió a la plantación de especies arbóreas y distintas plantas en el terreno escolar. No podemos olvidar que el $83 \%$ edificios que funcionaban en 1930 estaban emplazados en el medio rural y que 31 escuelas contaban con campos de experimentación agrícola.

Finalmente se describe la organización y el funcionamiento didáctico de estas escuelas: el plan de estudios; el personal docente, situado bajo la competencia de la Diputación, que al efecto estableció un detallado Reglamento de las Escuelas de Barriada, con amplio reconocimiento de derechos docentes que hasta el momento no se habían tenido en cuenta; el calendario escolar; los horarios oficiales, y la asistencia de los alumnos a clase, junto a otros detalles. El capítulo finaliza señalando otras actividades que se realizaban es estas escuelas, como las clases nocturnas para contribuir a erradicar el analfabetismo.

Aunque en la parte final se echan en falta datos e información ordenada sobre la desaparición de estas escuelas, la represión de los maestros y maestras y sus consecuencias, es evidente que analizando este proyecto podemos encontrar numerosos paralelismos con la situación actual. Las eskola-txikiak, escuelitas rurales actuales, son las que en cierta medida han tomado el relevo a esta experiencia innovadora. Para terminar, hay que destacar que este libro constituye una contribución importante para reivindicar la aportación de estas escuelas, así como para acercarnos a la historia de la educación e incluso a la historia social de Bizkaia.

Hilario Murua Cartón Universidad del País Vasco-Euskal Herriko Unibertsitatea hilario.murua@ehu.eus 